\title{
Research of the Photoelectric Tracking and Pointing Platform Based on the Servo Control System
}

\author{
Liu Shanzhong1, *, Han Pengna ${ }^{1}$
}

\author{
Liu S (1D https:/ / orcid.org/0000-0003-2486-6792 \\ Han P (D) https: / /orcid.org/0000-0002-8162-8267
}

How to cite

Liu S; Han P (2019) Research of the Photoelectric Tracking and Pointing Platform Based on the Servo Control System. J Aerosp Technol Manag, 11: e1419. https://doi.org/10.5028/jatm. $\underline{\mathrm{v} 11.1015}$

\begin{abstract}
To improve the speed and accuracy of the photoelectric tracking and pointing platform stable tracking, we took the three-axis photoelectric tracking platform, of which the coupling among the rings is ignored, as the research object. Considering the effects of various disturbances, the stable tracking double closed-loop servo control system was built for the azimuth, pitch, and roll rings. In the stabilization loop, output-feedback $\mathrm{H} \infty$ controller based on linear matrix inequality was designed to reduce the effects of model perturbations and uncertainties and to maintain the stability of the platform's inertial space. In the tracking loop, the integral-separation proportional integral derivative (PID) controller was designed to achieve fast and accurate tracking. Smith estimated compensation was adopted to compensate the pure lag caused by video tracking. Finally, the double closedloop servo control system was built to complete the simulation. The simulation results show that the system not only has good robustness under external disturbances, but also completes the attitude tracking quickly and accurately. Besides, the tracking error is $|e r r o r|<5 \cdot e^{-4}$ rad, which meets the tracking accuracy requirements.
\end{abstract}

KEYWORDS: Photoelectric tracking, Pointing platform, Servo control, Output-feedback $\mathrm{H} \infty$ control, Integral-separation PID.

\section{INTRODUCTION}

As a platform to achieve stable tracking and aim at the intended target, the photoelectric tracking and pointing platform is mainly composed of three parts, which are signal processing and control, a variety of photoelectric sensors, and stable tracking control (Huang et al. 2005; Ambrose et al. 2001). It's widely used in pods, guided weapons, and fire-control systems. The base of a photoelectric tracking platform is fixed on the moving platform, thus the photoelectric detection device can be easily affected by some uncertainties, such as the motion attitude of the ring, friction, vibration, noise, etc. This makes the video-axis of the detection device jitter and influences the extraction accuracy of the target-miss distance. Finally, the tracking performance of the whole system gets worse.

In the servo point of view, the photoelectric tracking and pointing platform is accurately tracking based on the optical axis stability. Hence, designing a stable controller with good robustness and high anti-interference ability is a prerequisite for achieving steady tracking. Domestic and foreign scholars have done a lot of researches. In literature (Hailiang 2013; Liu et al. 2008; Li et al. 2012), the stable controller was designed by using the linear quadratic Gaussian/loop transfer recovery (LQG/LTR) method, the self-organizing fuzzy control method, and the single-neuron adaptive proportional integral (PI) control method. These methods are robust and anti-interference to some extent. Srikasam et al. (2006) proposed a stable loop controller by using neural

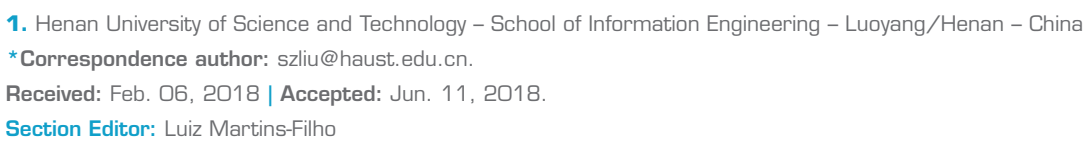


network-based optimal control algorithm, but the uncertainty disturbances can easily affect the selection of weights and fall into local optimum. $\mathrm{H} \infty$ control, an effective way for solving the perturbation and uncertainty disturbances, appeared in the model (Maher et al. 2014; Guo 2016). It's also available for multi-input and multi-output system (MIMO) and it has a good robustness (Huang et al. 2016). By solving the Riccati equation or the linear matrix inequality (LMI), documents (Michelin and Coustal 1991; Yang et al. 2010; Wang et al. 2010; Liu et al. 2016) get the status-feedback $\mathrm{H}_{\infty}$ controller and compare it with multi-input pole configuration method. The simulation shows that both have some robustness and that the status-feedback $\mathrm{H} \infty$ controller is easier to design. However, the state of an actual system usually cannot be measured directly, and the addition of a state observer will affect the stability of the system (Gu and Song 2007). This problem does not exist in the output-feedback. Therefore, under the premise of achieving stable control requirements, the output-feedback $\mathrm{H} \infty$ controller is a better choice.

In this work, we take the commonly used mathematical model in engineering practice, in which the coupling among the rings is ignored, as the research object. The main contribution of this paper is the design of an output-feedback $\mathrm{H} \infty$ stable controller to isolate all kinds of uncertain disturbances and to achieve stable control (the uncertain disturbances are assumed to be random but with limited energy). This control algorithm does not require fully observation of the system state, which is more reliable than the state-feedback $\mathrm{H} \infty$ control. In the tracking loop, the integral-separation proportional integral derivative (PID) control algorithm is used, whose tracking speed is fast and steady-state error is small, to achieve fast and accurate tracking.

\section{PRINCIPLE OF PLATFORM'S STABLE TRACKING}

The block diagram of the principle of stable tracking is shown in Fig. 1.

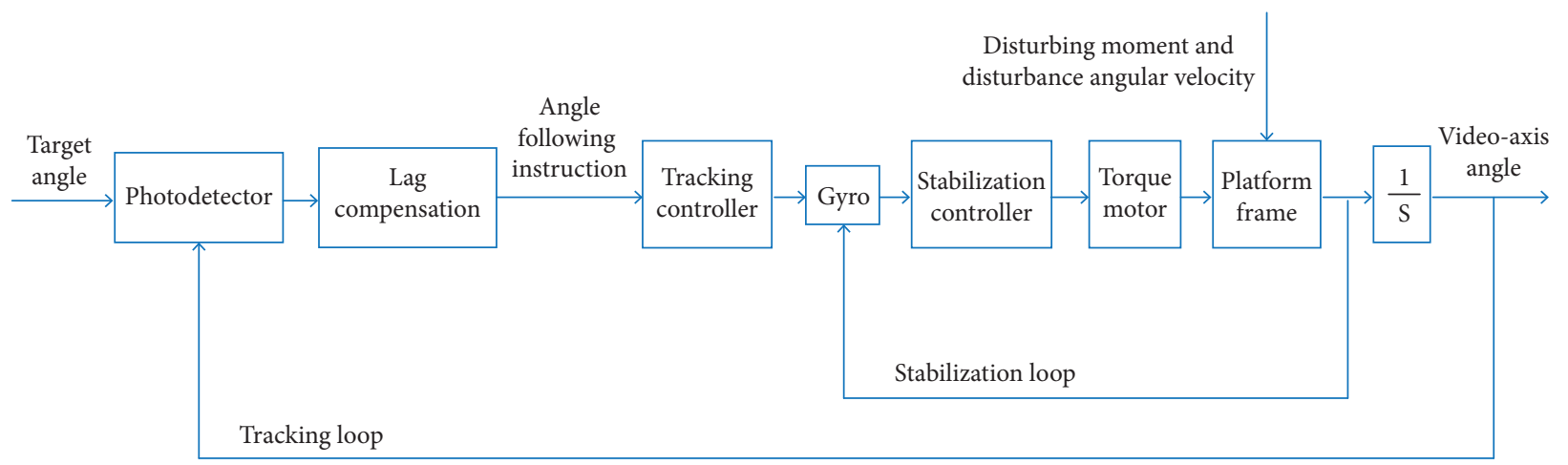

Figure 1. The principle of the platform's stable tracking.

From inside to outside, the rings of the tracking and pointing platform are the azimuth, pitch, and roll rings, successively (Cui and Huang 2007). The photodetector is attached to the azimuth ring. And the platform working in the tracking statement is an angular position servo system, the outer tracking loop, and the inner stabilization loop or the rate loop.

\section{ESTABLISHMENT OF MATHEMATICAL MODEL}

The stabilization loop of the photoelectric tracking and pointing platform consists of a platform frame seen as a controlled object, a power amplifier used to enlarge control instructions, a servo motor used to output drive torque, and a position gyro used to detect the platform's movement. The components of the pitch stabilization loop will be analyzed in the following part. 


\section{PLATFORM FRAME}

The mechanical structure of the platform, whose structure resonance frequency is generally believed to be higher than $200 \mathrm{~Hz}$, can be seen as rigid body in the low frequency signal. Hence, according to Newton's law of rigid body rotation, we can have Eq. 1:

$$
J_{\Sigma} \dot{\omega}=M_{\Sigma}
$$

where $J_{\Sigma}$ includes the motor rotor, which is connected to the platform frame, angle sensor rotor, and the sum of the frame itself at the moment of inertia (Tong and Liu 2014), $M_{\Sigma}$ includes the disturbance moment, which is resulted from the output torque and air-seat angular velocity and other disturbing torque, and $\omega$ is the angular velocity of the platform.

\section{SERVO TORQUE MOTOR}

We adopt the torque motor which is directly driven without decelerator. When the initial condition is zero, the armature circuit equation is given by Eq. 2 :

$$
i_{a}(s)=\frac{1}{R_{a}} \frac{U_{a}(s)-e(s)}{\tau_{\varepsilon} s+1}
$$

where $U_{a}, i_{a}$, and $R_{a}$ represent the output control voltage after the power amplifier, the armature current, and the resistance of the motor circuit, respectively. The $\tau_{\varepsilon}=L_{a} / R_{a}$ in the Eq. 2 is electromagnetic time constant, and $L_{a}$ is the inductance in the motor circuit.

The output torque of the motor is $M_{\mathrm{o}}(s)=K_{\mathrm{o}} i_{a}(s)$. Setting $\omega_{i}$ is the base disturbance angular velocity. The back-electromotive force $(\mathrm{EMF})$ of the servomotor is $e(s)=K_{\varepsilon}\left[\omega(s)-\omega_{i}(s)\right] . K_{\mathrm{o}}$ is torque coefficient, and $K_{\varepsilon}$ is back-EMF constant.

\section{POWER AMPLIFIER}

The pulse width modulation (PWM) mode was used for power amplification, which is generally regarded as the proportion of links.

\section{POSITION GYROSCOPE}

To improve the stable tracking accuracy of the system, we used a position gyro, whose transfer function is expressed as Eq. 3:

$$
G(s)=\frac{K_{m}}{s\left(\tau_{g} s+1\right)}
$$

where $\tau_{g}$ is time constant, $K_{\mathrm{m}}$ is scale coefficient, $K_{m}=K_{p} K_{a}$, the input of the gyroscope is the error between the command angular velocity $\omega_{a}$ and the angular velocity of the platform ring $\omega$, and the output is deviation angle.

\section{VIDEO IMAGE TRACKER}

Video tracker is the delay of the difference between the target line of sight and the platform video-axis. The transfer function is given by Eq. 4:

$$
G(s)=K e^{-\tau s}
$$

where $e^{-\tau s}$ is the part of delay and $\tau$ is delay time.

The scale coefficient is $K=$ angle of vertical field of view/number of scanning lines. Ignoring the coupling among the rings, taking the disturbing torque, which may affect the system parameters, and the disturbed angular velocity into account in the model parameter perturbation, and combining the mathematical model of the stabilization loop, we can get the space state model of the pitch ring's motion (Eq. 5). 
where

$$
A=\left[\begin{array}{cccc}
0 & \frac{1}{J_{\Sigma}} & 0 & 0 \\
-\frac{K_{\varepsilon} K_{o}}{\tau_{\varepsilon} R_{a}} & -\frac{1}{\tau_{\varepsilon}} & \frac{K_{o} K_{m}}{\tau_{\varepsilon} R_{a}} & 0 \\
0 & 0 & -\frac{1}{\tau_{g}} & \frac{1}{\tau_{g}} \\
-1 & 0 & 0 & 0
\end{array}\right], B_{1}=\left[\begin{array}{cc}
0 & \frac{1}{J_{\Sigma}} \\
1 & 0 \\
0 & 0 \\
0 & 0
\end{array}\right], B_{2}=\left[\begin{array}{llll}
0 & 0 & 0 & 1
\end{array}\right]^{\mathrm{T}}, C_{2}=\left[\begin{array}{llll}
1 & 0 & 0 & 0
\end{array}\right]
$$

The selected state variable is indicated by $x=\left[\begin{array}{llll}x_{1} & x_{2} & x_{3} & x_{4}\end{array}\right]$ where $x_{1}$ is the platform ring frame relative to angular velocity of inertial space, $x_{2}$ is the output torque of the motor, $x_{3}$ is the intermediate variable inside the gyroscope, $x_{4}$ is the deviation between the angular velocity of the instruction and the rotational angular velocity of the platform; $u$ is control input; and $w=\left[\omega_{i} M_{f}\right]^{\mathrm{T}}$ is disturbing input, where $\omega_{i}$ is disturbing angular velocity, and $M_{f}$ is disturbing torque.

The stable tracking system block diagram of the pitch ring is shown in Fig. 2.

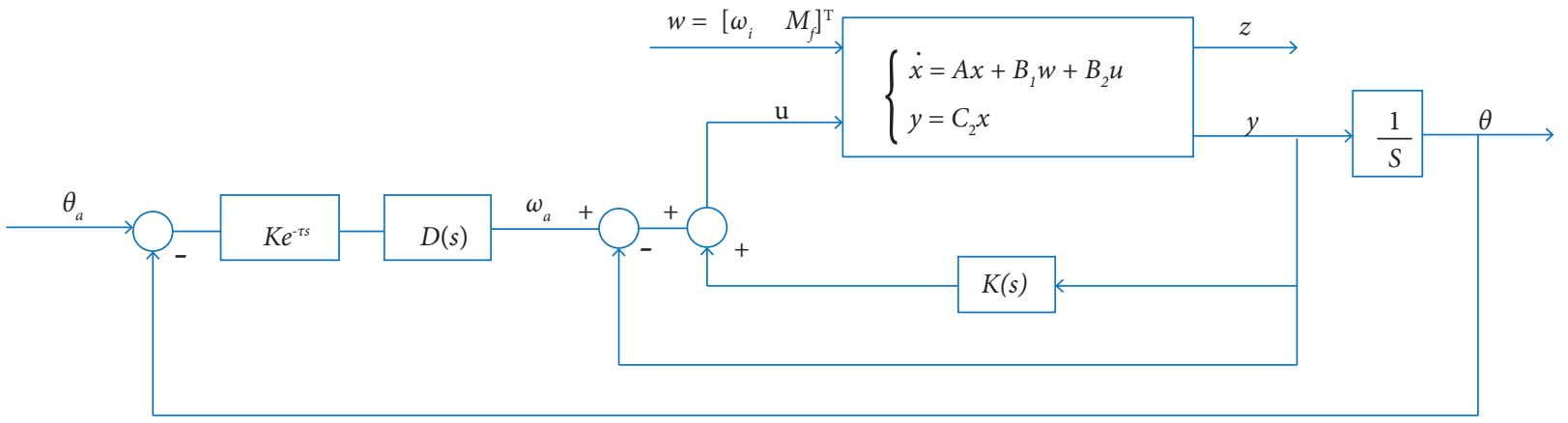

Figure 2. Stable tracking system structure.

where $\theta_{a}$ and $\theta$ are the target line of the sight angle and platform video-axis angle, respectively, $K(s)$ is the stable controller that we are going to design, and $D(s)$ is the tracking controller that we are going to design.

\section{STABILIZATION LOOP}

\section{THEORY OF OUTPUT-FEEDBACK H $\infty$ CONTROL BASED ON LMI}

Defining the performance evaluation index (Eq. 6):

$$
z=C_{1} x+D_{11} w+D_{12} u
$$

where $C_{1}, D_{11}$, and $D_{12}$ are the parameter matrices and $z$ is controllable output.

The state space model of the augmented controlled object of pitch loop is obtained (Eq. 7):

$$
\left\{\begin{array}{l}
x=A x+B_{1} w+B_{2} u \\
y=C_{2} x+D_{21} w+D_{22} u \\
z=C_{1} x+D_{11} w+D_{12} u
\end{array}\right.
$$


where $w$ is the disturbing signal.

Setting the disturbance is uncertain, but it has finite energy. That is $w \in L_{2}$.

The following indicators are set:

- $\quad\left(A, B_{2}\right)$ can stabilize and $\left(C_{2}, A\right)$ can be detected;

- $D_{11}=D_{22}=0$.

According to the state space form of the Eq. 7, an output-feedback $\mathrm{H} \infty$ controller $u=K(s) y(s)$ can be designed:

$$
\left\{\begin{array}{l}
\dot{\hat{x}}=A_{k} \hat{x}+B_{k} y \\
u=C_{k} \hat{x}+D_{k} y
\end{array}\right.
$$

where $\hat{x}$ is the state of controller $A_{k}, B_{k}, C_{k}$ and $D_{k}$ andare the parameter matrices of the controller.

Substituting Eq. 8 into Eq. 7 and $\xi=\left[\begin{array}{l}x \\ \hat{x}\end{array}\right]$, a closed-loop control system can be obtained:

$$
\left\{\begin{array}{l}
\dot{\xi}=A_{c 1} \xi+B_{c 1} w \\
z=C_{c 1} \xi+D_{c 1} w
\end{array}\right.
$$

where

$$
\begin{gathered}
A_{c l}=\left[\begin{array}{ll}
A+B_{2} D_{k} C_{2} & B_{2} C_{k} \\
B_{k} C_{2} & A_{k}
\end{array}\right] \quad B_{c l}=\left[\begin{array}{c}
B_{1}+B_{2} D_{k} D_{21} \\
B_{k} D_{21}
\end{array}\right] \\
C_{c l}=\left[\begin{array}{ll}
C_{1}+D_{12} D_{k} C_{2} & D_{12} C_{k}
\end{array}\right] D_{c l}=D_{11}+D_{12} D_{k} D_{21}
\end{gathered}
$$

It can be proved that the controller (Eq. 8) is the output-feedback $\mathrm{H} \infty$ controller of the system (Eq. 7). In other words, the closed-loop system (Eq. 9) is asymptotically stable. Aiming at the transfer function $T_{z w}$ from the disturbance input $w$ to the control output $z$, the following is the necessary and sufficient condition to satisfy $\left\|T_{z w}(s)\right\|_{\infty}<1$. If there is a positive definite symmetric matrix $X_{c l}$, such as that the matrix inequality Eq. 10 holds, then the output-feedback $\mathrm{H} \infty$ controller exists (Gu and Song 2007).

$$
\left[\begin{array}{ccc}
A_{c 1}^{\mathrm{T}} X_{c 1}+X_{c 1} A_{c 1} & X_{c 1} B_{c 1} & C_{c 1}^{\mathrm{T}} \\
B_{c 1}^{\mathrm{T}} X_{c 1} & -I & D_{c 1}^{\mathrm{T}} \\
C_{c 1} & D_{c 1} & -I
\end{array}\right]<0
$$

\section{DESIGN OF OUTPUT-FEEDBACK H $\infty$ CONTROLLER BASED ON LMI}

Based on the disturbance suppression performance index, order:

$$
C_{1}=\left[\begin{array}{cccc}
q_{1} & 0 & 0 & 0 \\
0 & q_{2} & 0 & 0 \\
0 & 0 & q_{3} & 0 \\
0 & 0 & 0 & q_{4} \\
0 & 0 & 0 & 0
\end{array}\right], D_{12}=\left[\begin{array}{c}
0 \\
0 \\
0 \\
r_{1}
\end{array}\right], D_{21}=\left[\begin{array}{ll}
r_{2} & 0
\end{array}\right]
$$


Firstly, the $q_{1}, q_{2}, q_{3}, q_{4}, r_{1}$ and $r_{2}$ parameters are reasonably selected to meet the setting targets. Then, the controller $K(s)$ is obtained by solving LMI and the $\mathrm{H} \infty$ performance index of the system is optimized by using mincx. In general, a suboptimal controller can be designed with $\mathrm{H} \infty$ performance, satisfying $\left\|T_{z w}(s)\right\|_{\infty}<\gamma$ to prevent the value of $K$ being too large and the mode of the controller being too fast. Finally, $A_{c 1}, B_{c 1}, C_{c 1}, D_{c 1}$ are substituted into Eq. 10 to verify whether it is established or not. If it is established, then $K(s)$ is the controller; if it is not established, the parameters in $C_{1}, D_{12}, D_{21}$ are adjusted and solved again.

Taking the design of the pitch stabilizing loop controller as an example, when $q_{1}=q_{2}=0.01, q_{3}=q_{4}=0.001, r_{1}=0.01$, and $r_{2}=0.1$, using MATLAB software, the suboptimal $\mathrm{H} \infty$ controller of $\left\|T_{z w}(s)\right\|_{\infty}<1$ can be obtained:

$$
\begin{gathered}
A_{k}=1.0 \mathrm{e}+04 \cdot\left[\begin{array}{cccc}
-0.2269 & 0.1647 & -0.0282 & 0.0834 \\
-0.0037 & -0.1489 & 0.0053 & -0.0130 \\
0.0918 & 0.6514 & -0.0148 & 0.0024 \\
0.3985 & 2.0861 & -0.0168 & -0.0276
\end{array}\right] \mathrm{B}_{k}=\left[\begin{array}{c}
-1.7224 \\
-0.8664 \\
41.9865 \\
-20.7395
\end{array}\right], \\
C_{k}=1.0 e+03 \cdot\left[\begin{array}{llll}
-2.4078 & 0.8393 & -0.2758 & 0.8496
\end{array}\right], D_{k}=-0.012
\end{gathered}
$$

The stability controller design of the azimuth and roll rings are similar to the above. We will not repeat them here.

\section{TRACKING LOOP}

\section{DESIGN OF INTEGRAL-SEPARATION PID ALGORITHM}

In the traditional PID control, the introduction of an integral part is mainly to eliminate static error and to improve control accuracy. But in the process of starting, ending, or in the presence of significant changes, the output of the system may present a great deviation in a short period of time set. This will cause integral accumulation and result in overshoot and even oscillation.

The following is the main idea of integral separation. When the system's error is large, the integral action should be canceled so that the system can respond quickly. This can also avoid the system's stability from getting poor and the overshoot from increasing at the same time. When the system's error is small, integral control is introduced to eliminate the static error and improve the control accuracy (Cheng et al. 2015; Huang et al. 2013). Its algorithm can be expressed as Eq. 11:

$$
\Delta \theta(k)=K_{p} \operatorname{error}(k)+\xi K_{I} \sum_{j=0}^{k} \operatorname{error}(j) T+\frac{K_{D}(\operatorname{error}(k)-\operatorname{error}(k-1))}{T}
$$

where $\xi$ is the switching coefficient of the integral term and $T$ is the sampling time.

$$
\xi=\left\{\begin{array}{l}
1,|\operatorname{error}(k)| \leq \varepsilon \\
0,|\operatorname{error}(k)|>\varepsilon
\end{array}\right.
$$

where $\varepsilon$ is threshold, which is set artificially.

The two input of integral-separation PID is the synchronization error. When $\varepsilon=0.1$ is selected from Eq. 12, the structure of integral-separation PID is shown in Fig. 3.

When $K_{p}=16, K_{1}=24$, and $K_{d}=0.01$ are taken in pitch tracking loop, good results can be obtained. 


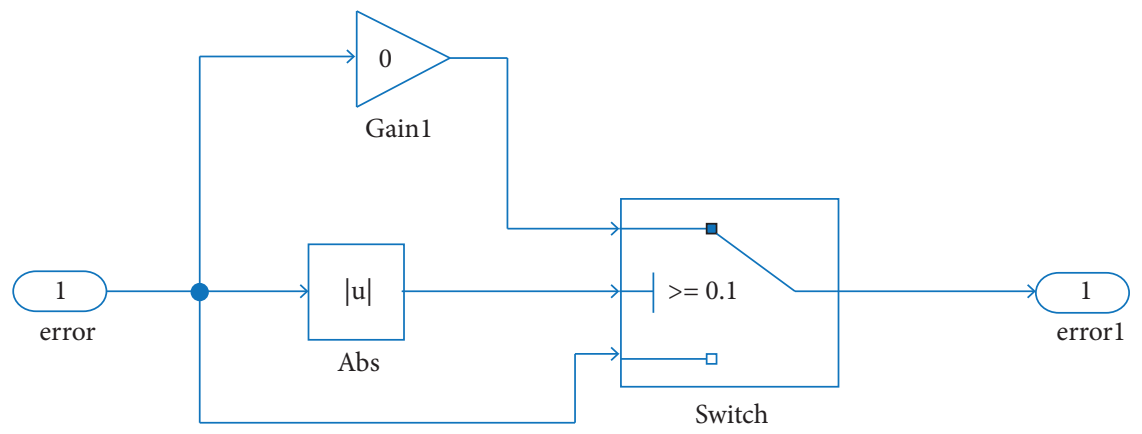

Figure 3. Integral-separation PID structure.

\section{DESIGN OF SMITH PREDICTIVE COMPENSATION}

The Smith predictor is used to eliminate the influence of the pure lag produced by the video tracker in the system and improve the dynamic performance of the system. Supposing that the transfer function of the controlled object with hysteresis is given by Eq. 13:

$$
G(s)=G_{1}(s) e^{-\tau s}
$$

where $G_{1}$ is the part without pure delay and $e^{-\tau s}$ is the pure delay part.

The following is the principle of Smith prediction compensation. By using a compensation link in parallel with $G(s)$, the pure hysteresis part is compensated. The transfer function of Smith predictor is designed as Eq. 14:

$$
\dot{G}=G_{1}(s)\left(1-e^{-\tau s}\right)
$$

where $\dot{G}(s)$ is the derivative of $G(s)$.

\section{SYSTEM ESTABLISHMENT AND SIMULATION RESEARCH}

In the stabilization loop, the output-feedback $\mathrm{H} \infty$ stabilization controller is designed for the azimuth, pitch, and roll rings. Then, its stability under external disturbances and parameter perturbations are analyzed. On this basis, the integralseparation PID algorithm is designed for the tracking outer loop. The Smith predictor is designed for the pure delay caused by the video tracking. Finally, the double closed-loop model is built, and the stable tracking control is simulated under the external disturbances.

\section{THE SIMULATION RESEARCH OF THE STABILIZATION LOOP}

(1) Suppose that the model parameters are determined, the square disturbance signals of $\omega_{i}=M_{f}$ are added to the azimuth, pitch, and roll rings. The scope of these disturbing signals are 0.02, 0.02, and 0.05, respectively, and all of their widths are 0.5 . Adding the air-seat stochastic angular vibration of frequency range from $20 \mathrm{~Hz}$ to $60 \mathrm{~Hz}$ at the same time, the simulation result of the stabilization loop is shown in Fig. 4.

(2) For the fact that the model parameter is usually uncertain in practice and that generally there is $10 \%$ of perturbation in the electric time constant of motor, the model parameter is usually uncertain. Taking $\tau_{\varepsilon}=(1+10 \%) \tau_{\varepsilon}$ as an example, when the disturbing signals are the same that in (1), the simulation result of the stabilization loop is shown in Fig. 5. 
Both Fig. 4 and Fig. 5 show that under the influence of external disturbances, whether the model parameter has perturbation or not, the simulation results are almost independent. It reflects that the output-feedback $\mathrm{H} \infty$ control is insensitive to model perturbation and uncertain disturbances and that it has good robustness.

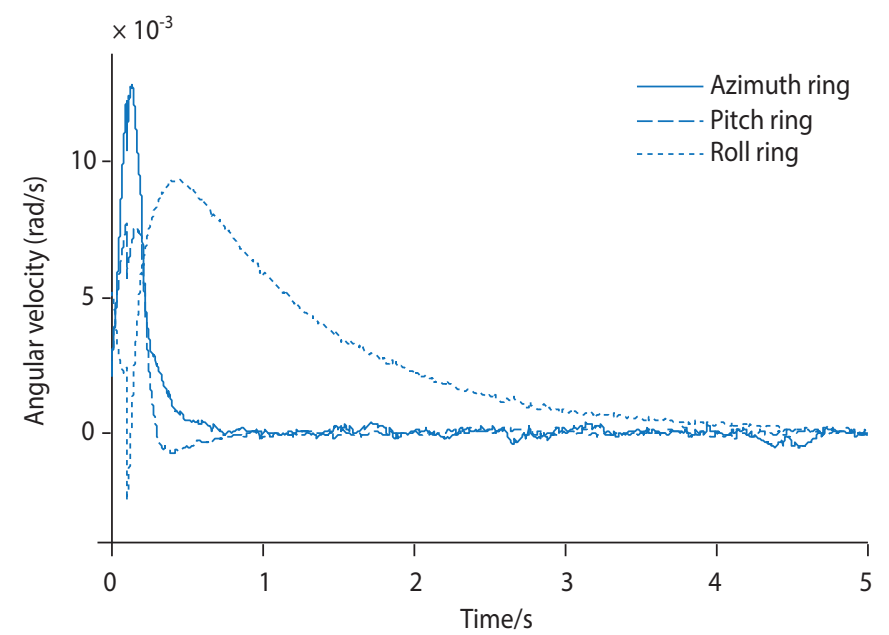

Figure 4. Parameter certainty and disturbances exiting response curve.

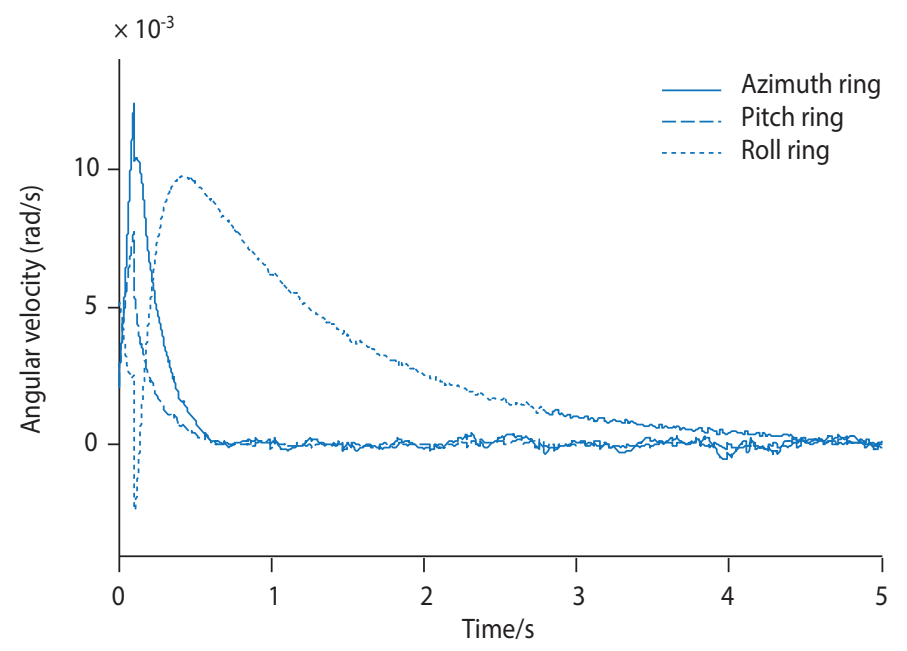

Figure 5. Simulation result of $\tau_{\varepsilon}=(1+10 \%) \tau_{\varepsilon}$.

\section{THE SIMULATION OF STABILIZATION AND TRACKING DOUBLE CLOSED-LOOP}

(3) The disturbing signals are the same as in (1). Based on output-feedback $\mathrm{H} \infty$ controller completion, the tracking effect of traditional PID is compared with the integral-separation PID algorithm in the tracking loop. When the three rings' control inputs are given by unit step signal, the output response curve is shown in Fig. 6.

The Fig. 6 shows that compared with the traditional PID, the tracking controller designed by integral-separation PID method not only reduces the overshoot of the system effectively, but also shortens the adjustment time. In the presence of various disturbances, fast and accurate tracking can be achieved.

(4) The disturbing signals are the same as in (1). In tracking loop, the integral-separation PID algorithm is adopted. When all of the three rings' control inputs are $\theta_{a}=0.2 t$, the simulation result of the attitude tracking control is shown in Fig. 7 , and the result of position tracking error is shown in Fig. 8. 


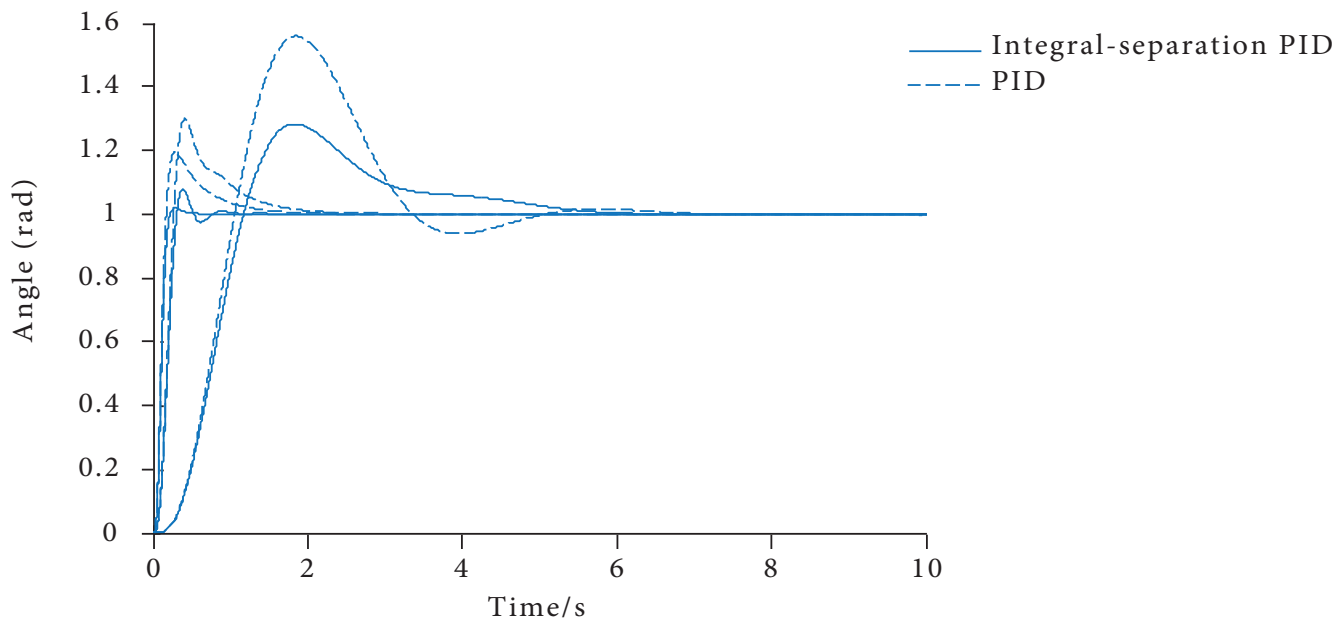

Figure 6. Tracking the comparison curve of step response.

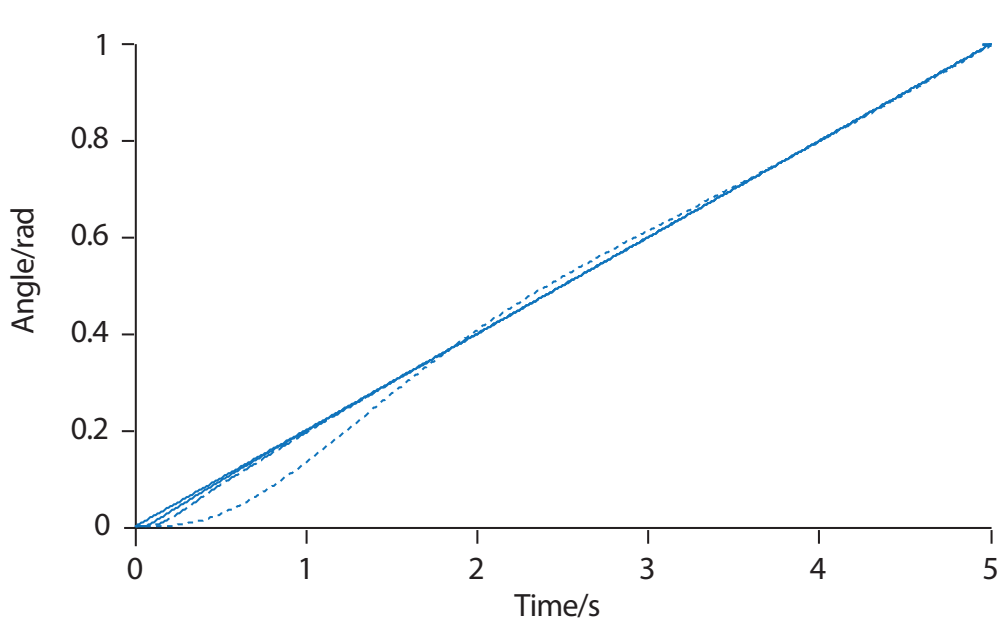

Ideal value

Azimuth ring

- - - - Pitch ring

Roll ring

Figure7. Simulation results of attitude tracking.

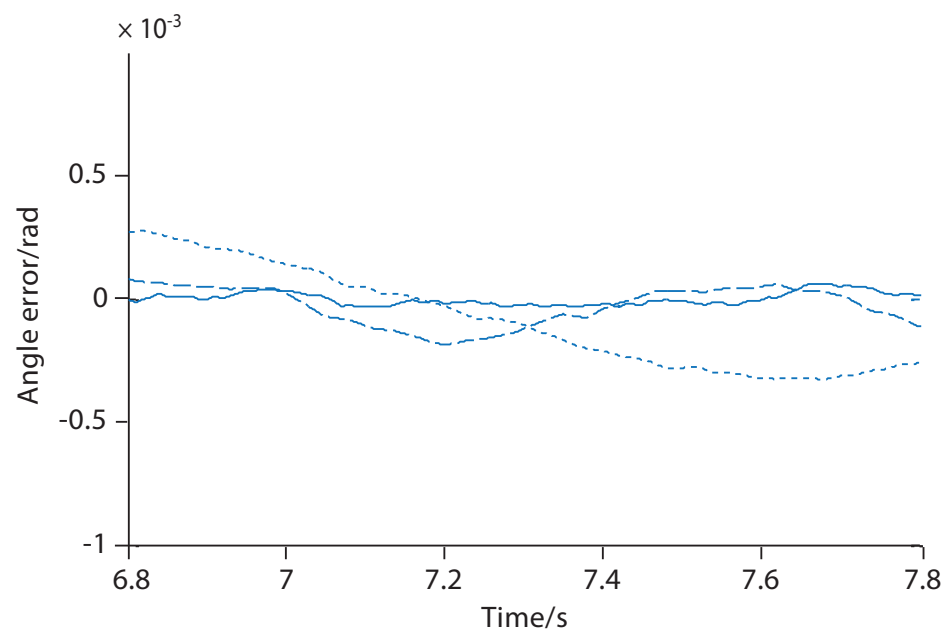

- Azimuth ring

- - - Pitch ring

-...- Roll ring

Figure 8. Position tracking error of three rings. 
Fig. 7 and Fig. 8 show that the three rings can also achieve fast and stable tracking when the control input changes. Furthermore, when the system is stable, the steady-state error is $\mid$ error $\mid<5 \cdot e^{-4} \mathrm{rad}$, which meets the tracking accuracy requirement (in general, tracking error less than $0.5 \mathrm{mrad}$ can meet the requirement of tracking accuracy).

\section{CONCLUSION}

We took the photoelectric tracking and pointing platform, of which the coupling among the rings is ignored, as research object. Firstly, in the stabilization loop, by selecting the appropriate weighting matrix, the design of output feedback H $\infty$ controller is completed for the azimuth, pitch, and roll rings. Simulation research shows that in the case of external disturbances and perturbation of the model parameter, the expected stabilizing control results are obtained with good robustness. Besides, the immeasurable state variables are not existing. Then, in the tracking loop, the integral-separation PID controller is designed. Finally, combining the speed loop controller, the simulation of stable tracking double closed-loop is completed. The simulation results show that the servo control system not only has strong anti-interference ability, but also creates stable and fast tracking control. And the tracking error meets the control precision requirement.

\section{FUNDERS}

National Natural Science Foundation of China [http://dx.doi.org/10.13039/50110001100]

Grant No: 61203047

\section{AUTHORS' CONTRIBUTION}

Methodology, Liu S and Han P; Building Platform and Simulation, Han P; Writing - Review, Han P; Simulation Analysis, Liu S and Han P; Supervision, Liu S.

\section{REFERENCES}

Ambrose H, Qu Z, Johnson R (2001) Nonlinear robust control for a passive line-of-sight stabilization system. Proceedings of the 2001 IEEE International Conference on Control Applications (CCA'O1) (Cat. No.01CH37204) p. 942-947. https://doi.org/10.1109/ CCA. 2001.973991

Cheng W-Y, Luo L, Liu Z (2015) Based on the Multi-axis Synchronous Sliding Mode Control of Integral Separation PID Algorithm Research. Modular Machine Tool \& Automatic Manufacturing Technique 8(8):97-100.

Cui L, Huang H (2007) Design and simulation of highly stable and precise tracking-pointing platform. Journal of Beijing University of Aeronautics and Astronautics 33(12):1462-1465.

Gu Q-Y, Song J-M (2007) Design of a missile attitude tracking controller based on $\mathrm{H} \infty$ robust output feedback. Acta Armamentarii 28(6):682-685

Guo S-X (2016) Robust reliability based optimal design of $\mathrm{H} \infty$ control of parametric uncertain systems. Journal of Dynamic Systems, Measurement, and Control 136(2):1-7. https://doi.org/10.1115/1.4025862

Hailiang Y (2013) The application of LQG/LTR method in turntable system. Harbin Institute of Technology p.55-65.

Huang J, Qian F, Xie G, Yang H (2016) Robust adaptive control for dynamic systems with mixed uncertainties. Journal of Systems Engineering and Electronics 27(3):656-663. https://doi.org/10.1109/jsee.2016.00069

Huang L, Zhao SH, Cui L (2013) Integral separation PID control in the control of lye concentration. Advanced Materials Research 627:489- 
493. https://doi.org/10.4028/www. scientific.net/amr.627.489

Huang Y, Tang X, Junfang LV (2005) Design and simulation of MIMO QFT stabilization controller of an airborne electro-optical tracking and pointing platform. Proceedings of the Gth International Conference on System Simulation and Science Computing; Beijing, China p. $1274-1278$.

Li Q, Zhu H-R, Gu J-P, Li J-H (2012) Single-neuron adaptive PI control of the gyrostabilized platform based on disturbance compensation. Electric Machines and Control 16(3):65-71,77.

Liu S-Z, Li Y-F, Liu Y-B, Qu X-M (2016) Stability controller design of electro-optical tracking and pointing platform. Fire Control \& Command Control 41(12):142-145.

Liu S-Z, Sun L, Che H (2008) Application of fuzzy control in electro-optical tracking and pointing platform stabilization control. Aerospace Control 26(2):55-58.

Maher RA, Mohammed IA, Ibraheem IK (2014) Polynomial based Ho robust governor for load frequency control in steam turbine power systems. International Journal of Electrical Power \& Energy Systems 57(5):311-317. https://doi.org/10.1016/i.ijepes.2013.12.010

Michelin J-M, Coustal P (1991) Control of a sight system flexible structure: A Ho design. Proceedings of the 3Oth IEEE Conference on Decision and Control 4:1640-1645. https://doi.org/10.1109/cdc.1991.261685

Srikasam W, Chaiyaratana N, Kuntanapreeda S (2006) Nonlinear system stabilisation by an evolutionary neural network. Lecture Notes in Computer Science p. 998-1006. https://doi.org/10.1007/11760023_147

Tong Z-J, Liu S-Z (2014) Fuzzy self-adaptive PID tracking control for airborne EO tracking and pointing platform. Electronics Optics \& Control 21(4):65-68.

Wang M, Zhao J, Dimirovski GM (2010) Output tracking control of nonlinear switched cascade systems using a variable structure control method. International Journal of Control 83(2):394-403. https://doi.org/10.1080/00207170903177766

Yang X, Gao H, Shi P, Duan G (2010) Robust Ho control for a class of uncertain mechanical systems. International Journal of Control 83(7): 1303-1324. 